\title{
What Causes the HI Holes in Gas-Rich LSB Dwarfs?
}

\author{
Katherine L. Rhode \\ Astronomy Department, Yale University, New Haven, CT 06520 \\ John J. Salzer \\ Astronomy Department, Wesleyan University, Middletown, CT 06459 \\ David J. Westpfahl \\ Department of Physics, New Mexico Institute of Mining \& Technology, \\ Socorro, NM 87801
}

\begin{abstract}
We have carried out a deep, multi-color imaging study of Holmberg II (Ho II) and several other nearby LSB dwarf galaxies for which detailed HI maps exist. The formation of the HI holes in these galaxies has been attributed to multiple supernovae ( $\mathrm{SNe}$ ) occurring within windblown shells around young, massive star clusters. To search for evidence of the clusters, we have compared optical images with the HI maps and measured magnitudes and colors of objects in and around the HI holes.

Although the SN scenario requires that detectable star clusters should often be present in the centers of the HI holes, our observations have in most cases failed to reveal these clusters at the expected magnitudes. In fact, many of the $\mathrm{HI}$ holes are located in regions of very low optical surface brightness, which show no evidence of recent star formation.
\end{abstract}

\section{Introduction}

Several nearby LSB dwarf galaxies have been mapped at high resolution in HI using the Very Large Array (Puche et al. 1992, Puche \& Westpfahl 1994). The maps show intricate structures in the galaxies' interstellar gas; analysis of the structures reveals many expanding $\mathrm{HI}$ holes. In the galaxy Ho II, for example, the holes are roughly spherical and surrounded by shells of higher density. Measured expansion velocities are typically $4-10 \mathrm{~km} \mathrm{~s}^{-1}$, and diameters are $100-1600 \mathrm{pc}$.

Puche et al. (1992; hereafter P92) proposed that the HI holes in Ho II and other dwarf galaxies were created by multiple, sequential SNe, occurring within wind-blown cavities in the interstellar medium (ISM). Using the expansion velocities and sizes of the holes, and the volume density of the surrounding medium, P92 calculated that many of the holes require the energy of $\sim 10-200 \mathrm{SNe}$ to create them, and that the holes typically have ages of $10^{7}-10^{8}$ years.

The SN scenario proposed by P92 provides us with a direct observational test. Multiple SNe are thought to occur in massive clusters or OB associations. The hole ages, if accurate, imply that the late-B, A, and F dwarf population 
should still be present in these clusters. For example, in a cluster which produces $50 \mathrm{SNe}$, a Salpeter IMF (Salpeter 1955) predicts 300 upper main sequence stars that should still be present after $10^{8}$ years. At the distance of HoII, the brightness of such a cluster would be $B \sim 22$, making it stand out against the low surface brightness level of the underlying older population of stars.

To search for the star clusters which would provide evidence for the SN scenario, we carried out a deep, multi-color optical imaging study of Ho II and the other dwarfs for which detailed HI maps exist. Our goal was to measure accurate magnitudes and colors of objects located in or near the HI holes. The published hole ages and energies can be used to calculate magnitudes and colors of the clusters that should be present if the SN scenario is correct. These predicted cluster properties can be directly compared to our observational data, to help refute or confirm the scenario described in P92.

\section{Observations and Photometry}

$B V R$ and $\mathrm{H} \alpha$ images of Ho II and $\sim 10$ other LSB dwarf galaxies were obtained in Feb 1994 and/or Apr 1995 with the 0.9-m telescope at Kitt Peak National Observatory. To create the deepest possible images with which to do photometry, the $B V R$ data from separate observing runs were merged. The HI maps from P92 and Puche \& Westpfahl (1994) were placed on the same pixel scale and aligned with our optical images to permit direct comparison.

Results from analysis of the data for Ho II are given here. Work on the other galaxies is still in progress. For Ho II, the information in P92 was used to mark the sizes and positions of the holes on the HI map and optical images. Photometry of our deepest $B V R$ frames was carried out at the locations of the hole and inter-hole regions. Photometry was carried out on 44 of the 51 hole regions identified in P92 (some HI hole locations coincided with foreground stars in the optical images, and had to be excluded from the sample). Twenty of these 44 holes had one or more faint objects located in or near the hole; photometry was executed on those objects individually, since they may be the star clusters which produced sequential SNe.

\section{Modelling the Putative Clusters}

To compare our observations with predictions arising from the SN scenario, we used the published hole properties to derive the observable properties of the clusters which should exist if the SN hypothesis is correct. Given the kinetic energy requirements for each hole, the number of SNe needed to create it was calculated. The energy imparted to the ISM by one supernova was taken to be $\sim 10^{51}$ ergs (McCray \& Kafatos 1987). For each hole, a Salpeter IMF (Salpeter 1955) was used to calculate the mass distribution for a model star cluster. This model cluster was scaled so that the number of stars with mass $\geq 7 \mathrm{M} \odot$ equalled the number of SNe required to create the hole. Stars that would leave the main sequence over a time period equal to the age of the hole were removed from the distribution. Finally, composite magnitudes and colors were calculated for the model cluster, for comparison with observations. A similar process was repeated using a Miller-Scalo IMF (Miller \& Scalo 1979), and assuming stars with mass 
$\geq 8 \mathrm{M} \odot$ become $\mathrm{SNe}$, to provide a reasonable range of predicted magnitudes and colors to compare with observations.

\section{Results and Conclusion}

Results for Ho II are summarized here; details may be found in Rhode et al. (1998):

- Many of the well-defined holes in the HI map of HoII are located in extremely LSB regions in the optical image, which show no evidence of recent star formation. Fourteen of the 44 holes in our sample lie beyond the Holmberg radius $\left(\mu_{B}=26.6 \mathrm{mag} \operatorname{arcsec}^{-2}\right)$, where star formation appears unlikely.

- There is no hint of a point source in 24 of the 44 holes in the sample. The $4 \sigma$-limit on the brightness of a point source in our data is $B=23$. Our models suggest that the remnant clusters which caused many of the holes should have $B \sim 21-22$; we would detect the clusters if they were present.

- We measured magnitudes and colors for 29 sources in the 20/44 holes which had objects located within the hole or just outside it. Ten of the sources have $B-V>1.0$, thereby excluding them as likely candidates for the putative clusters.

- A total of 12 holes, in the main optical body of the galaxy, contain objects that could be the putative clusters; i.e., the objects have magnitudes and colors that may be consistent with a massive young cluster. We cannot rule out the $S N$ hypothesis for those 12 HI holes. Note, however, that such holes appear in regions of relatively high galaxian background, which makes it difficult to determine whether the objects that appear to coincide with them are actually associated with a given hole.

The observational evidence strongly suggests that at least some of the HI holes in Ho II did not originate in precisely the manner envisioned by P92. A number of alternative explanations for the origin of the holes have been put forth, including the recent suggestion that the holes are remnants of Gamma-Ray Burst events (Efremov et al. 1998; Loeb \& Perna 1998). These alternative explanations, and how they relate to our findings, are discussed thoroughly in Rhode et al. (1998).

\section{References}

Efremov, Y.N., Elmegreen, B.G., \& Hodge, P.W. 1998, ApJ, 501, L163

Loeb, A., \& Perna, R. 1998, ApJ, 503, L35

McCray, R., \& Kafatos, M. 1987, ApJ, 317, 190

Miller, G.E., \& Scalo, J.M. 1979, ApJS, 41, 513

Puche, D., \& Westpfahl, D. 1994, in Proceedings of the ESO/OHP Workshop on Dwarf Galaxies, eds. G. Meylan \& P. Prugniel (ESO: Garching), 273

Puche, D., Westpfahl, D., Brinks, E., \& Roy, J.-R. 1992, AJ, 103, 1841

Rhode, K.L., Salzer, J.J., Westpfahl, D.J., \& Radice, L.A. 1998, AJ, submitted Salpeter, E.E. 1955, ApJ, 121, 161 